\title{
Vesícula biliar retrohepática hipotrófica, intrahepática, hepático derecho accesorio aberrante. Reporte de caso y revisión de la literatura
}

\author{
Hypotrophic, retrohepatic and intrahepatic gallbladder with aberrant right \\ hepatic duct. A case report and literature review
}

Héctor Bizueto-Rosas, ${ }^{*}$ Iván Hernández-Altamirano, ${ }^{* *}$ Juan de Dios Pérez-Reyna, *** Marco Antonio Romero-López, ${ }^{* * *}$ Norma Cruz-Sánchez, ${ }^{*}$ Daniela Oropeza-Adame****

\begin{abstract}
Palabras clave:
Vías biliares,

variantes anatómicas,

hepático derecho

accesorio, cirugía.
\end{abstract}

Key words: Biliary tract, anatomical variants, accessory hepatic right duct, surgery.

\section{RESUMEN}

La formación embriológica compleja de la vesícula y árbol biliar es la responsable de las frecuentes variantes anatómicas en más de $50 \%$ de los casos, de las cuales en su mayoría no ocasionan patología alguna. De las variantes anatómicas, las anomalías de los conductos derechos son las que se presentan con mayor frecuencia respecto a las vías biliares. Los divertículos vesiculares y la desembocadura en la vesícula biliar de un conducto hepático derecho accesorio son subtipos bastante raros. Presentamos el caso de un paciente masculino de 42 años con múltiples episodios de cólico biliar; en el último se intervino de urgencia y se documentaron estas variantes anatómicas poco frecuentes, como hallazgo incidental. Posteriormente se realizó colangiopancreatografía transduodenal retrógrada endoscópica que demostró integridad de la vía biliar y ausencia de litiasis residual. Por lo anterior concluimos que la colangiografía intraoperatoria debería realizarse de rutina, tanto para descartar litiasis coledociana como para detectar variantes anatómicas, pues de su detección depende el pronóstico transoperatorio, postoperatorio y la vida del paciente. Presentamos una revisión de la literatura sobre estas anomalías.

\section{ABSTRACT}

The embryological complexity in the formation of the gallbladder and biliary tract are responsible for the frequent anatomical variants in more than $50 \%$ of the cases, the majority of which do not cause any pathology. In the anatomical variants, abnormalities in the right duct are the most frequent regarding biliary tract. The gallbladder diverticulum and accessory right hepatic duct entering the gallbladder are quite rare. We present the case of a male patient of 42 years with multiple episodes of biliary pain; in the last episode required urgent surgery and those anatomical variants were observed. Later on, an endoscopic retrograde transduodenal cholangiopancreatography demonstrated the integrity of the bile duct and absence of residual lithiasis. Therefore, we conclude that the intra-operative cholangiography should be routine for both discard lithiasis as to detect anatomical variations, because recognition of those impacts prognosis in the transoperative period, postoperative and the patient's life. We present a review of the literature about these anomalies.

\footnotetext{
* Médico adscrito de Cirugía General.

** Residente del $4^{\circ}$ año de Cirugía General. ** Residente del $3^{\circ}$ año de Cirugía General. $* * * *$ Residente del $2^{\circ}$ año de Cirugía General.
}

Hospital General "Dr. Darío Fernández Fierro". Instituto de Seguridad y Servicios Sociales de los Trabajadores del Estado. México, Distrito Federal.

Recibido: 15/06/2015 Aceptado: 30/05/2016

\section{INTRODUCCIÓN}

Ela n la cuarta semana de gestación el hígado, la vesícula biliar y la vía biliar se originan a partir de una yema sólida en forma de un rudimento situado en la pared ventral del intestino anterior primitivo. ${ }^{1}$ Esta yema se divide en una porción craneal sólida del divertículo hepático (pars hepatis) que forma el tejido hepático glandular, los conductos hepáticos intrahepáticos y la porción caudal (pars cystica), la vesícula biliar, conducto cístico, el colédoco y el páncreas ventral.

La porción distal del conducto hepático se divide en una rama derecha y una rama izquierda, las cuales se canalizan por vacuolización. ${ }^{1}$

El pedículo de la vesícula biliar se convierte en el conducto cístico. El pedículo que une los conductos hepáticos y cístico al duodeno se convierte en el conducto colédoco. ${ }^{1}$ Este 
complicado proceso de embriogénesis origina las variantes anatómicas frecuentes, las cuales en su mayoría no ocasionan patología alguna, por lo que a menudo son hallazgos incidentales durante los procedimientos quirúrgicos o endoscópicos. ${ }^{1}$

Se han reportado variantes durante las disecciones quirúrgicas en $28 \%$ de los casos y de 5 a $13 \%$ en las colangiografías transoperatorias. $13 \%$ de los individuos presentan variaciones en los conductos biliares, incluyendo variaciones en el conducto cístico, colédoco o conductos hepáticos accesorios. ${ }^{1}$

La anatomía de los conductos biliares sigue la segmentación del sistema porta en el hígado. Sólo la mitad presenta la llamada "anatomía normal" con variantes entre 24 y $57 \%{ }^{2}$

Las variaciones de la vía biliar principal poseen una explicación embriológica y se relacionan con una teoría hemodinámica de la segmentación hepática, lo que implica una mayor variación anatómica de los conductos derechos. ${ }^{1}$

La vesícula biliar, saco en forma de pera de 7 a $10 \mathrm{~cm}$ de largo, con una capacidad promedio de 30 a $50 \mathrm{ml}$ se localiza en la superficie visceral del hígado en una fosa profunda, dividiendo el lóbulo hepático derecho del segmento medio del lóbulo izquierdo, normalmente en la unión de los segmentos 4 y 5.

Las variantes anatómicas de la vesícula biliar se presentan en $40 \%$ de los casos; intrahepática (5-10\%), flotante (existencia de un mesenterio largo), en el lóbulo izquierdo del hígado; ${ }^{3}$ rudimentaria o hipoplásica en forma de reloj de arena; doble o múltiple (0.02-0.5\%) y agenesia vesicular en $0.03-0.1 \% .{ }^{1,4}$

La vesícula en gorro frigio es la anomalía más común. ${ }^{5}$ Los divertículos vesiculares son poco frecuentes, se localizan en cualquier parte de la vesícula. La vesícula biliar rudimentaria y la vesícula en reloj de arena son congénitas en niños y adultos, adquiridas por fibrosis por cuadros repetitivos de colecistitis en adultos.

Estas anomalías son irrelevantes clínicamente y asintomáticas, salvo que se acompañen de otra patología asociada. ${ }^{6}$

El conducto hepático que desemboca en la vesícula es raro, por lo que el conducto cístico se continúa como colédoco, predisponiendo a la lesión del conducto hepático. ${ }^{1}$ Los conductos accesorios persistentes "fetal" entre el hígado y la vesícula existen en $8.7-28 \% .7,8$

El conducto hepático derecho accesorio se presenta en $5 \%$ de los pacientes. Los conductos accesorios generalmente pequeños, atraviesan el triángulo de Calot y se unen al conducto hepático común o desembocan en la vesícula (Figura 1). Ocasionalmente se unen al cístico. Pueden ser el único drenaje de segmentos del hígado derecho (conductos aberrantes); puede realizarse una colangiografía a través del conducto para conocer la porción de hígado afectado y la extensión y saber si estamos ante un conducto accesorio o aberrante. Strasberg clasifica la lesión a estas estructuras como tipo B y C. ${ }^{4}$

Las manifestaciones clínicas están en relación con el calibre del conducto y el territorio hepático afectado; si el diámetro del conducto lesionado mide más de $3 \mathrm{~mm}$, debe restablecer su continuidad mediante un asa en $Y$ de Roux. ${ }^{4}$

El conducto cístico es el elemento anatómico más importante desde el punto de vista quirúrgico, pues nos permite la realización de ciertas maniobras, como la colangiografía transoperatoria, entre otras; en consecuencia al tener la ubicación precisa, aunque no siempre es posible definir bien la vía biliar, disminuye el riesgo transoperatorio de lesiones.

En los momentos cruciales del procedimiento quirúrgico debemos considerar las variantes anatómicas del cístico (desembocadura anómala 30\%). ${ }^{1}$ Goor clasifica 11 tipos de anomalías en la terminación del conducto cístico. Puede duplicarse o estar ausente, por lo que el cuello vesicular se abre directamente en la vía biliar principal. ${ }^{8}$ El conocimiento de estas posibles variantes anatómicas es esencial en la prevención de lesiones de la vía biliar. ${ }^{1}$

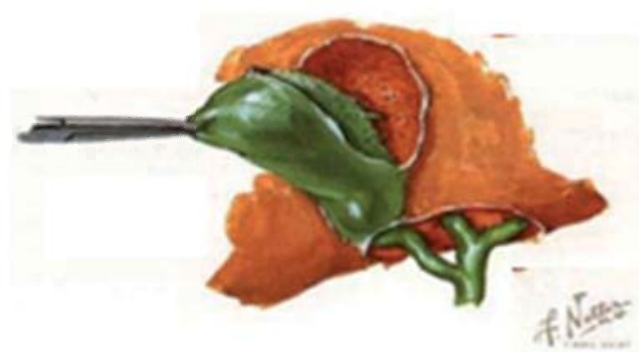

Figura 1. Conducto hepático derecho accesorio. Tomada de: Boleko-Ribas MA. p.17. ${ }^{4}$ 
La colangiografía transoperatoria es el arma principal para orientarnos sobre la vía biliar y sus variantes anatómicas, ${ }^{9-11}$ no siempre es posible realizarla; los cirujanos ya no tienen medio de contraste por estar más familiarizados con el abordaje laparoscópico o por cuestiones administrativas de las unidades médicas. ${ }^{9-11}$

Los conductos accesorios de Luschka o subvesicales miden por lo general de 1 a 2 mm y se originan del lóbulo hepático derecho. Pueden ser únicos o existir como una red de conductillos. Drenan al conducto hepático derecho, al hepático común y rara vez al hepático izquierdo. No drenan ninguna región en particular del hígado y no se acompañan de la tríada portal. ${ }^{4}$

La ligadura de un hepático accesorio puede cursar con fiebre e ictericia hasta en 15\% que tienden a ser pasajeras y sin mayor relevancia clínica.

No obstante que ahora disponemos de recursos clínicos y tecnológicos para llegar a un diagnóstico que nos permita realizar la elección del tratamiento más adecuado de la patología biliar, no siempre puede llevarse a cabo, pues el ultrasonido es operador-dependiente y el paciente con dolor abdominal agudo por lo general cursa con íleo reflejo que ocasiona distensión abdominal considerable, aunado a la obesidad, las variantes anatómicas de la vesícula y vías biliares o las formas de presentación clínica.

La colecistitis aguda conlleva a la lesión de la vía biliar, aunque algunos autores opinan que facilita el plano de disección por el edema existente. Ooi observó que la inflamación en el triángulo de Calot era un factor de riesgo asociado a la lesión de la vía biliar en la edad avanzada. Se ha mencionado, y nosotros lo hemos corroborado, que la patología vesicular en el hombre conlleva más dificultad quirúrgica, no sólo por el ángulo cerrado entre el borde costal, la línea media y la masa muscular, sino porque en el cuadro agudo es frecuente encontrar reacción inflamatoria severa con fibrosis considerable. ${ }^{12}$

Presentamos el caso de un paciente masculino con un conducto hepático accesorio que desemboca en una vesícula hipotrófica y de localización anómala.

\section{PRESENTACIÓN DEL CASO}

Masculino en la quinta década de la vida, sin antecedentes de importancia, con dolor abdominal en hipocondrio derecho, se le diagnosticó por ultrasonografía colecistitis aguda litiásica sin coluria ni acolia, fue intervenido encontrando vesícula hipotrófica de 3 $\mathrm{cm}$ aproximadamente por debajo del proceso caudado y no en la superficie visceral del hígado, invaginación intrahepática en su porción infundibular de alrededor de $2 \times 1 \mathrm{~cm}$ con litos en su interior, adherida firmemente al conducto hepático común. En la cara hepática de la vesícula biliar desembocaba un conducto de 1.5 $\mathrm{mm}$, el cuerpo vesicular corría paralelamente al conducto colédoco con adherencias firmes y fibrosas (Figura 2).

En la cara posterior del lóbulo caudado se observó una prominencia de $2.5 \times 2 \mathrm{~cm}$ que correspondía a la vesícula biliar intrahepática. Se trató de resecar para identificar el cuello de la vesícula; sin embargo, estaba firmemente adherida y empezó a sangrar, por lo que desistimos continuar con el procedimiento.

Al no contar con colangiografía transoperatoria, se decidió ligar el conducto que desembocaba en la vesícula y resecar ésta última con técnica retrógrada, subtotal, para identificar por dentro su desembocadura en la vía biliar; se ocluyó la parte que corresponde a su ostium a $2 \mathrm{~mm}$ de la vía biliar con sutura de poliglactina $3(0)$.

Se colocó un drenaje mixto (sonda nelaton $24 \mathrm{Fr}$ multiperforada con camisa de Penrose de $\left.0.5^{\prime \prime}\right)$ al lecho vesicular y un Penrose a la corredera parietocólica derecha.

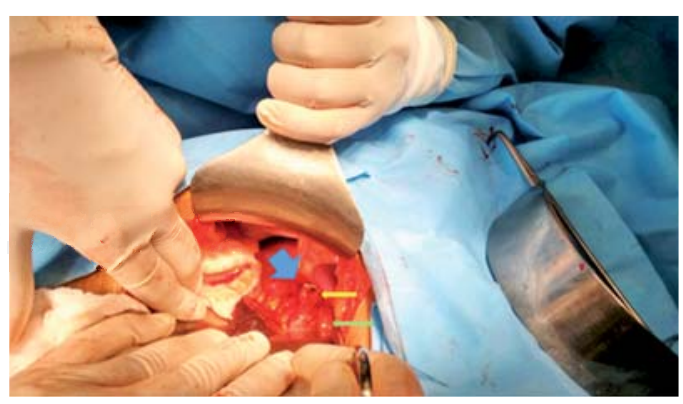

Figura 2. Flecha azul hepático accesorio desemboca en la vesícula, flecha amarilla divertículo vesicular, flecha verde colédoco. 
Posteriormente, a las 48 horas se realizó colangiografía transduodenoscópica sin evidenciar lesión a la vía biliar, litiasis residual o fuga biliar.

El estudio histopatológico no demostró ningún proceso maligno.

\section{DISCUSIÓN}

Anatomía biliar. Tolino, et al.: mencionan que Nari, et al. encontraron La anatomía normal, Tipo A de Blumgart sólo en aproximadamente la mitad de los casos. ${ }^{2}$

Grey Turner: "Las lesiones de vía biliar desafortunadamente no son raras y con frecuencia se convierten en tragedias". ${ }^{13}$

Maingot: "La colecistectomía fácil y un cirujano carente de adecuado entrenamiento quirúrgico constituyen una combinación siniestra". ${ }^{14}$

Bismuth: "Lo más relevante es saber evitar estas lesiones, en caso de que se produzcan hay que saber repararlas y manejar las complicaciones." 4

"Cualquier cirujano puede tener una lesión de la vía biliar tras una colecistectomía sin que esto se considere mala praxis".

"Todavía hay, y seguirán existiendo, cirujanos en desarrollo, aprendiendo, que deben ser supervisados adecuadamente; la curva de aprendizaje es personal".

"Una lesión de la vía biliar debe seguir representando una alarma".

La mayor parte de las lesiones de la vía biliar se deben a mala interpretación de la anatomía, así como a la presencia de variantes anatómicas. ${ }^{4}$

Las variantes, frecuentemente inocuas, son hallazgos incidentales durante la cirugía o endoscopias. Se han encontrado variantes del sistema biliar en $2.4 \%$ de las necropsias, $28 \%$ en la cirugía y en $5-13 \%$ de las colangiografías transoperatorias. Por lo que tener en cuenta estas posibles variaciones de la anatomía biliar es crucial en la prevención de lesiones de la vía biliar. ${ }^{1}$

La colangiografía retrógrada endoscópica (CRE) es indispensable en el diagnóstico de la patología de vías biliares. Sin embargo, no está exenta de complicaciones (de 5 a 10\%), con riesgo de infección de 0.6 a 5\%, hemorragia de
0.3 a $2 \%$, perforación de 0.1 a $1.1 \%$, pancreatitis aguda de 1.3 a $6.7 \%$ y mortalidad de 0.02 a $0.5 \% .{ }^{1}$ Actualmente sólo se recomienda su uso para fines terapéuticos.

No se ha demostrado relación entre el riesgo de lesión biliar y las variaciones anatómicas del pedículo biliar. La mayoría de las lesiones biliares aparecen durante colecistectomías que no presentan especial dificultad o las que se realizan durante el cuadro agudo de colecistitis aguda, puesto que la inflamación del triángulo de Calot es un factor de riesgo importante asociado a la lesión de la vía biliar. ${ }^{12}$

En nuestro caso, al resecar parcialmente la vesícula y poder identificar el cístico por dentro se constató que se trataba de un cístico corto o incluso ausente al visualizar únicamente el ostium de la vesícula.

La mayoría de las lesiones durante la colecistectomía se producen al confundir la vía biliar principal con el conducto cístico, aunado a la presencia de un conducto cístico corto. ${ }^{4,14}$

El proceso inflamatorio severo, las adherencias entre la vesícula y el duodeno, su localización posterior e intrahepática impidieron la visualización correcta del área y pudo haberse pasado inadvertido un quiste del colédoco o haberse confundido el divertículo de la vesícula intrahepática con un quiste del hepático derecho con las consecuencias de lesión (quiste de Todani tipo IV).

En las vesículas intrahepáticas el riesgo de complicaciones es mayor por el sangrado, por tal motivo se optó por no resecar completamente la vesícula.

Los divertículos vesiculares son poco frecuentes y se localizan en cualquier parte de la vesícula. No tienen mayor importancia clínica, son asintomáticos, salvo que se acompañen de otra patología asociada.

En 10\% existe un conducto hepático derecho accesorio, raro, pero es la más frecuente de las variantes anatómicas que favorece la lesión del colédoco.

Simeone y cols. distinguen los conductos hepáticos accesorios de los aberrantes. Los primeros proveen de un drenaje secundario a una región hepática determinada, en cambio los conductos aberrantes representan la única vía de drenaje de una región hepática que se ubica en una localización inusual. ${ }^{1}$ 
En caso de tratarse de un conducto de Luschka, su detección intraoperatoria es improbable; la colangiografía intraoperatoria tampoco lo detecta. La ligadura o el clipaje son suficientes para el tratamiento de dichas fugas, ya que estos conductos no drenan ninguna parte significativa de parénquima hepático. ${ }^{4}$

Moosa valoró las circunstancias de iatrogenia de las vías biliares en 81 pacientes, encontró diversos factores de riesgo como acceso inadecuado, exposición, iluminación deficiente, carencia de ayudantes, ausencia de colangiografía, obesidad, disección inadecuada del triángulo de Calot, colecistitis aguda, sangrado y variantes anatómicas arteriales. ${ }^{13,15}$

"Primero, evitar estas lesiones, saber repararlas y manejar las complicaciones" ${ }^{4}$ De lo contrario, debemos colocar drenajes y referir a un centro con mayor experiencia.

En nuestra unidad no contamos con equipo para realizar colangiografía transoperatoria a nuestro paciente masculino ${ }^{12}$ que presenta vesícula intrahepática, conducto hepático accesorio, ausencia de conducto cístico, divertículo vesicular, localización anómala de la vesícula, proceso inflamatorio y fibroso severo.

\section{CONCLUSIONES}

No en vano existen las descripciones detalladas de la vía biliar, pues es probable que las variantes ocasionen complicaciones catastróficas que pueden terminar con la vida de un paciente e influir en el pronóstico transoperatorio y postoperatorio.

La colangiografía transoperatoria es de vital importancia y debería llevarse a cabo de manera rutinaria para evitar complicaciones, puesto que el beneficio de su realización es estadísticamente mayor que el riesgo. ${ }^{16}$

Es posible ligar la lesión de conductos segmentarios o subsegmentarios menores de 3 $\mathrm{mm}$; los conductos mayores de $4 \mathrm{~mm}$ deberán repararse. ${ }^{13,15,17}$

Es necesario reparar la vía biliar cuando la lesión se detecta intraoperatoriamente. Si no se cuenta con la experiencia suficiente, habría que colocar un drenaje en el lecho quirúrgico y derivar al paciente a un centro de referencia donde reciba un tratamiento multidisciplinario. ${ }^{4}$

\section{Agradecimiento}

Al Dr. Hugo Alonso Pérez González, residente de Angiología, por su asistencia técnica.

\section{REFERENCIAS}

1. Aguirre-Olmedo I, Fernández-Castro E, GonzálezAngulo JA, Cárdenas-Lailson LE, Beristain-Hernández JL. Variantes anatómicas de la vía biliar por colangiografía endoscópica. Rev Gastroenterol Mex. 2011; 76: 330-338.

2. Tolino MJ, Tartaglione AS, Sturletti CD, García MI. Anatomy variations of biliary tree. Surgical implicance. Int J Morphol. 2010; 28: 1235-1240.

3. Rothman M. Anomalías de la vesícula y de las vías biliares y de sus vasos sanguíneos. En: Berk JE, editor. Bockus HL Gastroenterología. Tomo VI: Vías biliares y páncreas. 6⿳a ed. España: Salvat; 1987, p. 780-781.

4. Boleko-Ribas MA. Estudio retrospectivo de las lesiones iatrogénicas de la vía biliar durante la era laparoscópica en un hospital de tercer nivel. [Tesis doctoral]. Barcelona: Hospital Universitari Vall d'Hebron, Servei de Cirurgia General i de l' Aparell Digestiu; 2011.

5. Pérez-Torres E, Espino-Cortés $\mathrm{H}$, Bernal-Sahagún F. Vesícula biliar doble. Informe de un caso. Rev Med Hosp Gen Mex. 2004; 67: 94-97.

6. Cueva-Pacheco A. Vesícula biliar. Departamento Académico de Cirugía, editores. Cirugía General I. [Libro en Internet]. Lima Perú. Disponible en: http:// sisbib.unmsm.edu.pe/bibvirtual/libros/medicina/cirugía.

7. Sinnatamby Ch S. Hígado y vías biliares. En: Last RJ. Anatomía de Last, regional y aplicada. $10^{a}$ ed. Barcelona: Paidotribo; 2003, p. 259-60.

8. Kurumi Y, Tani T, Hanasawa K, Kodama M. The prevention of bile duct injury during laparoscopic cholecystectomy from the point of view of anatomic variation. Surg Laparosc Endosc Percutan Tech. 2000; 10: 192-199.

9. Ponsky JL. Complications of laparoscopic cholecystectomy. Am J Surg. 1991; 161: 393-395.

10. Raute M, Schaupp W. latrogenic bile duct injuries at cholecystectomy. Management and results. Langenbeck's Arch Chir. 1988; 373: 345-354..

11. Sackier JM, Berci G, Paz-Partlow M. Elective diagnostic laparoscopy. Am J Surg. 1991; 161: 326-331.

12. Vergnaud J, Penagos S, Lopera C, Herrera A, Zerrate A, Vasquez J. Colecistectomia laparoscópica: experiencia en hospital de segundo nivel. Rev Colomb Cir. 2000; 15: 8-13.

13. Mercado-Díaz MA, Ramírez-Morales R, MedinillaCruz MA, Sánchez Medal FP. Férula transhepáticatransanastomótica en lesiones de las vías biliares. Evolución a largo plazo. Cir Ciruj. 2008; 76: 219-223.

14. Matthews JB. Blumgart LH. Estenosis biliares benignas. En: Zinner MJ, Schwartz SI, Ellis H, editores. Vol II: Maingot. Operaciones Abdominales. 10a ed. Buenos Aires: Ed. Panamericana; 1998, pp. 1691-721.

15. Melton GB, Lillemoe KD, The current management of postoperative bile duct strictures. Adv Surg. 2002; 36: 193-221. 
16. Menéndez A, Kleinfinger S, Vidal P, Ramírez C, Kunz W, González M. Riesgo-beneficio de la colangiografía transoperatoria de rutina. Rev Mex Cir Endoscop. 2004; 5: 99-103.

17. Contreras-Sibaja E, Rendon-Dosal HD. Aspectos demográficos en lesión de vía biliar en un periodo de 8 meses en colecistectomía laparoscópica en el Hospital Central Militar. Rev Sanid Milit Mex. 2015; 69: 393-398.
Correspondencia:

Dr. Héctor Bizueto Rosas

Puerto Zihuatanejo Núm. 18,

Col. Ampliación Casas Alemán,

Del. Gustavo A Madero, 07580,

Ciudad de México, México.

Tel. (55) 35990728

E-mail:dr_bizueto_h@yahoo.com 